\title{
Feasibility and acceptability of combining cognitive behavioural therapy techniques with swallowing therapy in head and neck cancer dysphagia
}

\author{
J. M. Patterson ${ }^{1,2^{*}}$, M. Fay, C. Exley ${ }^{3}$, E. McColl ${ }^{1}$, M. Breckons ${ }^{1}$ and V. Deary ${ }^{4}$
}

\begin{abstract}
Background: Head and neck cancer squamous cell carcinoma (HNSSC) patients report substantial rates of clinically significant depression and/or anxiety, with dysphagia being a predictor of distress and poorer quality of life. Evidence-based dysphagia interventions largely focus on the remediation of physical impairment. This feasibility study evaluates an intervention which simultaneously uses a psychological therapy approach combined with swallowing impairment rehabilitation.

Methods: This prospective single cohort mixed-methods study, recruited HNSCC patients with dysphagia, from two institutions. The intervention combined Cognitive Behavioural Therapy with swallowing therapy (CB-EST), was individually tailored, for up to 10 sessions and delivered by a speech and language therapist. Primary acceptability and feasibility measures included recruitment and retention rates, data completion, intervention fidelity and the responsiveness of candidate outcome measures. Measures included a swallowing questionnaire (MDADI), EORTCQLQH\&N35, dietary restrictions scale, fatigue and function scales and the Hospital Anxiety and Depression Scale (HADS), administered pre-, post-CB-EST with three month follow-up and analysed using repeated measures ANOVA. Qualitative interviews were conducted to evaluate intervention processes.
\end{abstract}

Results: A total of 30/43 (70\%) eligible patients agreed to participate and 25 completed the intervention. 84\% were male, mean age 59 yrs. Patients were between 1 and 60 months (median 4) post-cancer treatment. All patients had advanced stage disease, treated with surgery and radiotherapy (38\%) or primary chemoradiotherapy (62\%). Pre to post CB-EST data showed improvements in MDADI scores $(p=0.002)$, EORTC-QLQH\&N35 $(p=0.006)$, dietary scale $(p<0.0001)$, fatigue $(p=0.002)$ but no change in function scales or HADS. Barriers to recruitment were the ability to attend regular appointments and patient suitability or openness to a psychological-based intervention.

Conclusions: CB-EST is a complex and novel intervention, addressing the emotional, behavioural and cognitive components of dysphagia alongside physical impairment. Preliminary results are promising. Further research is required to evaluate efficacy and effectiveness.

\footnotetext{
* Correspondence: joanne.patterson@ncl.ac.uk

${ }^{1}$ Institute of Health and Society, Newcastle University, The Baddiley-Clark

Building, Richardson Road, Newcastle upon Tyne NE2 4AX, UK

${ }^{2}$ Speech \& Language Therapy Department, Sunderland Royal Hospital,

Sunderland, UK

Full list of author information is available at the end of the article
} 


\section{Background}

Chronic swallowing difficulties (dysphagia) are a common and highly distressing side effect of surgery and/or (chemo)radiotherapy for the treatment of head and neck cancer (HNSCC) [1]. Dysphagia is associated with a higher risk of pneumonia, poor oral intake, malnutrition and prolonged tube feeding [2]. HNSSC patients report substantial rates of clinically significant depression and/ or anxiety, with dysphagia being a predictor of distress $[3,4]$. Our previous qualitative work reported on fundamental changes to eating habits, social lives and wellbeing, with some patients being better able to adjust to such changes than others [5].

Evidence based HNSCC dysphagia interventions largely centre on impairment-focused treatments delivered by speech \& language therapists (SLTs) [6]. These typically include exercises to increase the range and co-ordination of swallowing function, to improve efficiency and safety. These may be administered before (as a preventative approach), during or after HNSCC treatment. The degree to which exercises prevent or reduce dysphagia is unclear due to poor patient adherence and differing exercise protocols [7]. Patients need support in coping with side effects [8], but to date, there are minimal reports of interventions addressing the psychosocial sequelae of dysphagia.

General psychosocial interventions for HNSCC patients such as psycho-education, counselling and cognitive behavioural therapy (CBT) have been reported. A Cochrane review concluded that the subsequent impact on quality of life of these interventions was uncertain due to study design limitations [9]. Whether a psychological-based treatment can be combined with impairment-based swallowing therapy to address dysphagia is an unknown. Potentially this type of intervention could improve patient engagement with rehabilitation and facilitate adjustment in living with swallowing difficulties. CBT has previously been used with HNSCC [10] and has been used by SLTs for other conditions [11].

This study aims to investigate the feasibility and acceptability of a cognitive behavioural enhanced swallowing therapy (CB-EST) for HNSCC dysphagia, using a mixed methods design.

\section{Methods}

This is a multi-centre, prospective, longitudinal nonrandomised single cohort study to explore feasibility and acceptability of a CBT enhanced swallowing therapy intervention (CB-EST) in HNSCC patients. The feasibility design was informed by guidance set out by the CONSORT 2010 statement [12].

\section{Patients and eligibility}

HNSCC patients were recruited from two units in NE England. They were eligible for the study if they 1) had completed HNSCC treatment with curative intent 2) were medically stable and 3) scored $<80$ points on the MD Anderson Dysphagia Inventory [13] (swallowing specific quality of life questionnaire). Patients were excluded if they 1) had pre-existing major psychiatric diagnosis 2) had residual/recurrent HNSCC 3) were on a palliative care pathway 4) had significant communication difficulties rendering them unable to participate in a talking therapy 5) were currently receiving a psychological intervention 6) were awaiting an intervention for the purpose of improving swallowing performance (e.g. a dilatation) or 7) had significant ill-health precluding regular hospital attendance. Patients were screened and approached by members of the multi-disciplinary HNSCCC team and gave written consent before participation. The study aimed to recruit thirty participants, to provide data to perform a sample size calculation for a potential future effectiveness study [14].

\section{Intervention}

The main researcher (JP), a SLT trained in CBT to post graduate certificate level, delivered the intervention. CBEST was individually tailored, but aimed to include key CBT components i.e. in-depth assessment, identification of maintaining factors within a formulation, identification of a therapy goal, Socratic questioning style, cognitive and/or behavioural therapy techniques and homework tasks. The intervention also included individualised swallowing exercises, diet modifications and food texture advice, if appropriate to the patient's therapy goal. Between 45 and 60 min were allowed for each session. Treatment was on a weekly or fortnightly basis (depending on patient preference and need for support) for up to 10 sessions by mutual agreement, with a follow up assessment at three months to monitor generalisation and maintenance. JP received supervision from an expert CBT practitioner every $2-3$ weeks.

\section{Feasibility outcomes}

As this was primarily a feasibility study, primary outcomes were those that related to the acceptability of the intervention to participants and the feasibility of trialling the intervention in a larger study. The acceptability and feasibility outcomes are as follows:

1. Acceptability was measured by the proportion of patients approached and consented and the number of sessions attended. Retention rates and reasons for drop out was documented. We aimed for a $50 \%$ recruitment rate for CB-EST to be deemed an acceptable treatment.

2. Feasibility and fidelity were measured by assessing whether the intervention could be delivered as planned, by a SLT with CBT training. Session 
content and treatment plans, recorded in patients' notes were evaluated by a CBT expert practitioner as part of supervision, reliability and validity checking. Content analysis of sessions including a) whether a therapy goal was identified b) whether a CBT formulation was identified c) whether cognitive and/or behaviour change techniques were used. These outcomes would also indicate the acceptability of the intervention to patients.

3. A selection of candidate measures targeting swallowing self-report, dietary restrictions, quality of life, functioning and mood were chosen to identify appropriate tools to capture CB-EST outcomes. Acceptability to patients was monitored by percentage data completion. The measures listed below and were administered pre-, immediately following CBEST, and at three months.

i. The MDADI [13] has twenty items, each marked using a five-point scale and summarised using a total score (range 20-100). Higher scores indicate a better outcome and a change in $\geq 10$ points is considered a clinically significant difference [15].

ii. The European Organization for Research and Treatment of Cancer questionnaires (EORTC QLQ-C30) [16] is a general quality of life questionnaire with 30 items, five functioning scales (physical, role, emotional, cognitive, and social), three symptom scales. The EORTC QLQ-H\&N35 is a disease-specific module of 35 questions divided into 7 subscales about pain, swallowing, senses, speech, social eating, social contact, and sexuality. Higher scores on the functional scales refer to better health status, whereas higher scores in symptom scales and the QLQ-H\&N35 represent more severe symptoms.

iii. Chalder Fatigue Questionnaire (CFQ-11) [17] measures fatigue severity. Eleven items are answered on a four-point scale (range $0-33$ ), with high scores representing more fatigue.

iv. Work and Social Adjustment Scale(WASA) [18] measures functional and social impairment. Five questions are answered on a nine-point scale (range 0-40) with higher scores indicating more impairment.

v. Hospital Anxiety and Depression Scale (HADS) [19] has two seven item subscales measuring anxiety (HADS-A) and depression (HADS-D). Each item is scored on a four-point scale (range 0-21 for each subscale). Subscale scores 0-7 classify participants as non-cases, 8-10 indicates borderline cases, and scores $\geq 11$ indicate clinical levels. Total HADS scores (HADS-T) $\geq 15$ indicate clinically significant distress. vi. Performance Status Scales (PSS) Normalcy of Diet [20] measures diet texture restrictions and is clinician-rated. The scale has ten ranked categories ranging from 0 (nil by mouth) to 100 (full diet without restrictions).

The presence of a feeding tube was recorded at the same time points. The sensitivity of the candidate measures was tested by making preliminary estimates of change from pre- to post CB-EST. Data were analysed using SPSS v21 (Chicago, Illinois). We used a one way within subjects repeated measures analysis of variance complete case model. The level for statistical significance was set at 0.05 . Bonferroni's test was used for multiple post hoc comparisons. Means are reported with standard deviations and 95\% confidence intervals.

4. The acceptability and feasibility of delivering CB-EST as-was or modifying it for a larger trial was further assessed using semi-structured interviews. Patients were purposively sampled to ensure a range of pre to post CB-EST changes in MDADI scores, a range of HNSCC treatment and time posttreatment. Patients were selected from those at the initial stages of CB-EST and at the end of CB-EST. Interviews were conducted by two independent researchers. Patients had the option of a telephone or face to face interview, at a time and place of their choice. All interviews were digitally recorded, $t$ ranscribed verbatim and anonymised. Transcripts were read several times and in detail by the qualitative sub-team. Data were then discussed and coded using thematic analysis. Quotations relating to afore mentioned topics were independently selected and coded into key issues and themes.

\section{Ethics}

Ethical approval was granted by the UK North East Research Ethics Committee reference 14/NE/1045.

\section{Results Feasibility and acceptability as measured by recruitment and retention}

Fifty patients were screened over 20 months. Seven patients reported that their eating and drinking issues had resolved and/or they scored $>80$ on the MDADI, so were ineligible. Forty-three patients were approached and 30 gave written consent (69.8\%). Patient characteristics for consented patients are summarised in Table 1. There was no statistical difference in distribution of gender, age, disease site, stage, type of treatment or time since treatment $(p>0.05)$ between those that consented to participation and those that did not. 
Table 1 Patient baseline characteristics and demographics for consented CB-EST patients

\begin{tabular}{|c|c|}
\hline Characteristics & Number (\%) \\
\hline \multicolumn{2}{|l|}{ Gender } \\
\hline Male & $26(87)$ \\
\hline Female & $4(13)$ \\
\hline Age & 59 (range 49-79) \\
\hline \multicolumn{2}{|l|}{ Disease site } \\
\hline Oropharynx & $18(60)$ \\
\hline Oral & $5(17)$ \\
\hline Nasopharynx & $3(10)$ \\
\hline Larynx & $2(7)$ \\
\hline Hypopharynx & $1(3)$ \\
\hline Unknown primary & $1(3)$ \\
\hline \multicolumn{2}{|l|}{ Stage } \\
\hline 0 & $1(3)$ \\
\hline 1 & $2(6)$ \\
\hline 2 & $13(43)$ \\
\hline 3 & $7(24)$ \\
\hline 4 & $7(24)$ \\
\hline \multicolumn{2}{|l|}{ Nodes } \\
\hline 0 & $3(10)$ \\
\hline 1 & $7(24)$ \\
\hline 2 & $20(66)$ \\
\hline \multicolumn{2}{|l|}{ Treatment } \\
\hline Chemoradiotherapy & $19(63)$ \\
\hline $\begin{array}{l}\text { Surgery and radiotherapy } \\
+/- \text { chemotherapy }\end{array}$ & $9(30)$ \\
\hline Surgery & $1(3.5)$ \\
\hline Radiotherapy & $1(3.5)$ \\
\hline Time post-treatment (months) & Median 4 (IQR, Range 3,13; 1-60) \\
\hline \multirow[t]{2}{*}{ Partner } & Yes (23) \\
\hline & No $(7)$ \\
\hline
\end{tabular}

Reasons for non-participation included difficulties with regular hospital attendance (5), did not think CB-EST would help (3), did not attend (2), currently receiving CBT (1), and too much to take on (2). Twenty five patients were retained $(83 \%)$ in the intervention. Three patients dropped out at sessions 3,4 and 5 due to disease (2 local disease, 1 lung cancer). Two patients opted not to continue with CB-EST at session 2 and 5; one found it difficult to envisage how CB-EST might be of benefit and the other felt he was making insufficient progress. No outcome data were available for drop-outs. The number of CB-EST sessions for the retained patients ranged from 3 to 10 sessions, median 6. At three month follow up, one patient was too unwell to complete questionnaires.
Feasibility and acceptability as measured by intervention fidelity

All retained patients were able to identify a goal specific to their eating and drinking problem. Goals fell into six main areas (see Table 2). A formulation for all but one drop-out patient was developed and verified during supervision. Examples of formulations are provided in Fig. 1. A range of cognitive and behavioural techniques were utilised during CB-EST and are recorded in Table 2.

\section{Acceptability and utility of candidate outcome measures} Data completeness ranged from 76 to $100 \%$, the PSS Normalcy of diet scale having the highest compliance, QLQ-C30 having the lowest compliance (see Table 3). Post CB-EST improvements were observed in several measures. Although not powered for effectiveness, a statistically significant improvement was observed in MDADI scores $(p=0.002$, mean difference 8.1$)$; three domains of QLQ-C30 function scales ( $p=0.004-0.03$ mean difference 10.4-21.3); seven domains on QLQHN35 symptoms scales $(p<0.0001-0.01$ mean difference 10.8-14.7); CFS ( $\mathrm{p}=0.002$ mean difference 4.1) and PSS Normalcy of diet scale $(\mathrm{p}<0.0001$ mean difference 15.8). These improvements were maintained at three months. No statistical improvement was observed for two domains on QLQ-C30 functioning scales; two domains on QLQ-HN35 symptom scales; WASA scales or the HADS (see Table 3).

\section{Feasibility and acceptability as measured by participant interviews}

Sixteen patients were approached for interview and 15 consented. The interviewer was unable to arrange a convenient time for three patients. There were ten telephone and two face to face interviews. One patient opted to be interviewed with her partner present. The sample reflected baseline characteristics for gender, age, site and stage of disease (see Table 4).

Three main CB-EST process themes were identified.

\section{Duration and frequency}

Participants were referred to CB-EST either by a specialist nurse or a SLT and all felt this was an appropriate pathway. Overall, patients were happy with the duration of sessions. Session length of approximately one hour was deemed sufficient to explore issues and to decide on homework until the following session. Generally, patients felt that between 8 and 10 sessions was an appropriate set of meetings. Some felt that regular intervals (weekly/fortnightly) were beneficial whilst others would have preferred them to be more frequent in the early stages of CB-EST. 


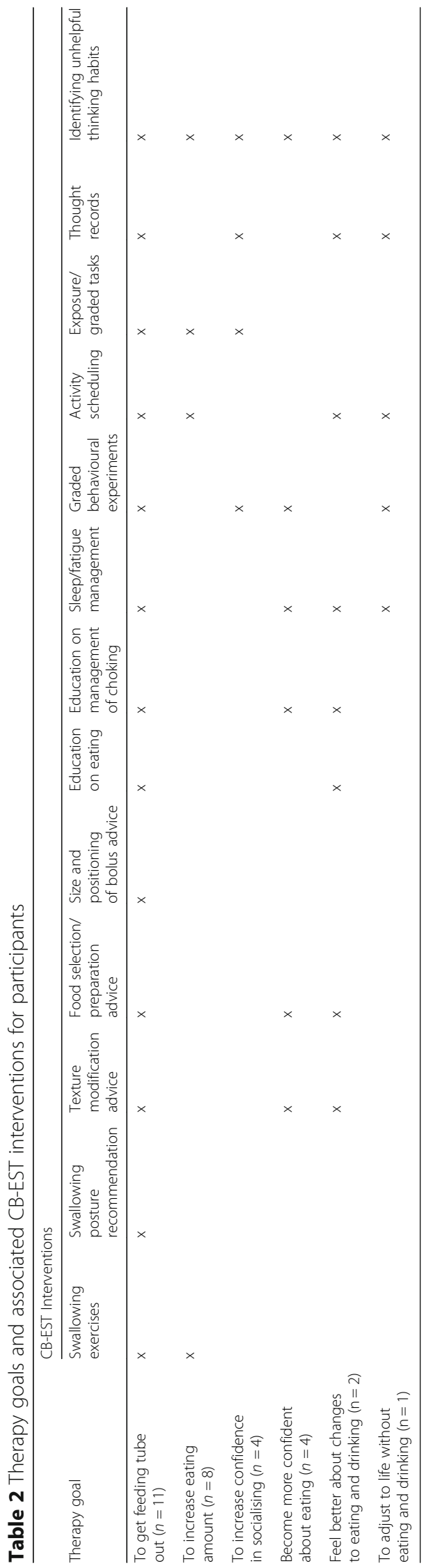




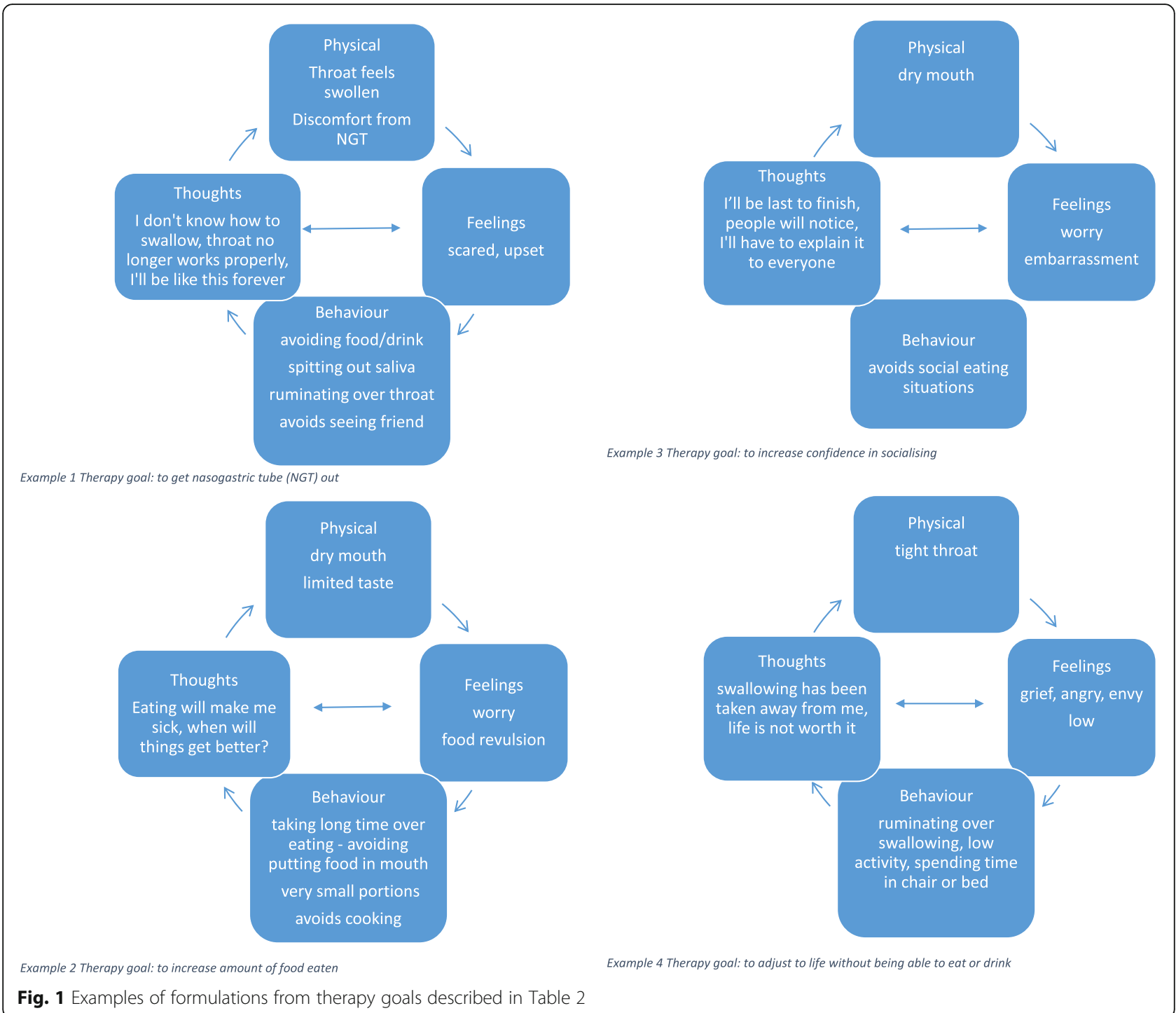

'The timeframe between that was just balanced nicely and allowed me to kind of make those plans and have an experience to then come back and talk about it, so yeah it worked well' (S3 aged $51 \mathrm{yrs})$.

Participants understood that sessions were a finite resource and moving on was an inevitable and necessary part of the intervention. Several participants stated that they would have happily continued as they discovered an overall benefit on recovery and well-being. Patients liked the combination of talking, visualising issues using a whiteboard and other applied techniques, and concrete goal setting.

\section{Timing}

Most participants felt that the timing of their participation in CB-EST worked well, with some expressing that they wished they had earlier access.
'Maybe it should be part of the initial journey and treatment ... not everybody might take it but at least it is there isn't it' (S7 aged 61 years).

There was variation about whether there is an ideal time point to start sessions. Timing seemed to be related to individual preferences and symptom severity. Participants seem to fall roughly into two groups: those who would prefer the sessions to accompany their treatment (i.e. start soon after diagnosis) and those who thought sessions are more beneficial following (but not too long after) treatment.

\section{Suitability}

Some participants described themselves as being suited to an intervention such as CB-EST, i.e. either being open to talk about emotions and/or relatively open to help and change. Others described themselves as being more 


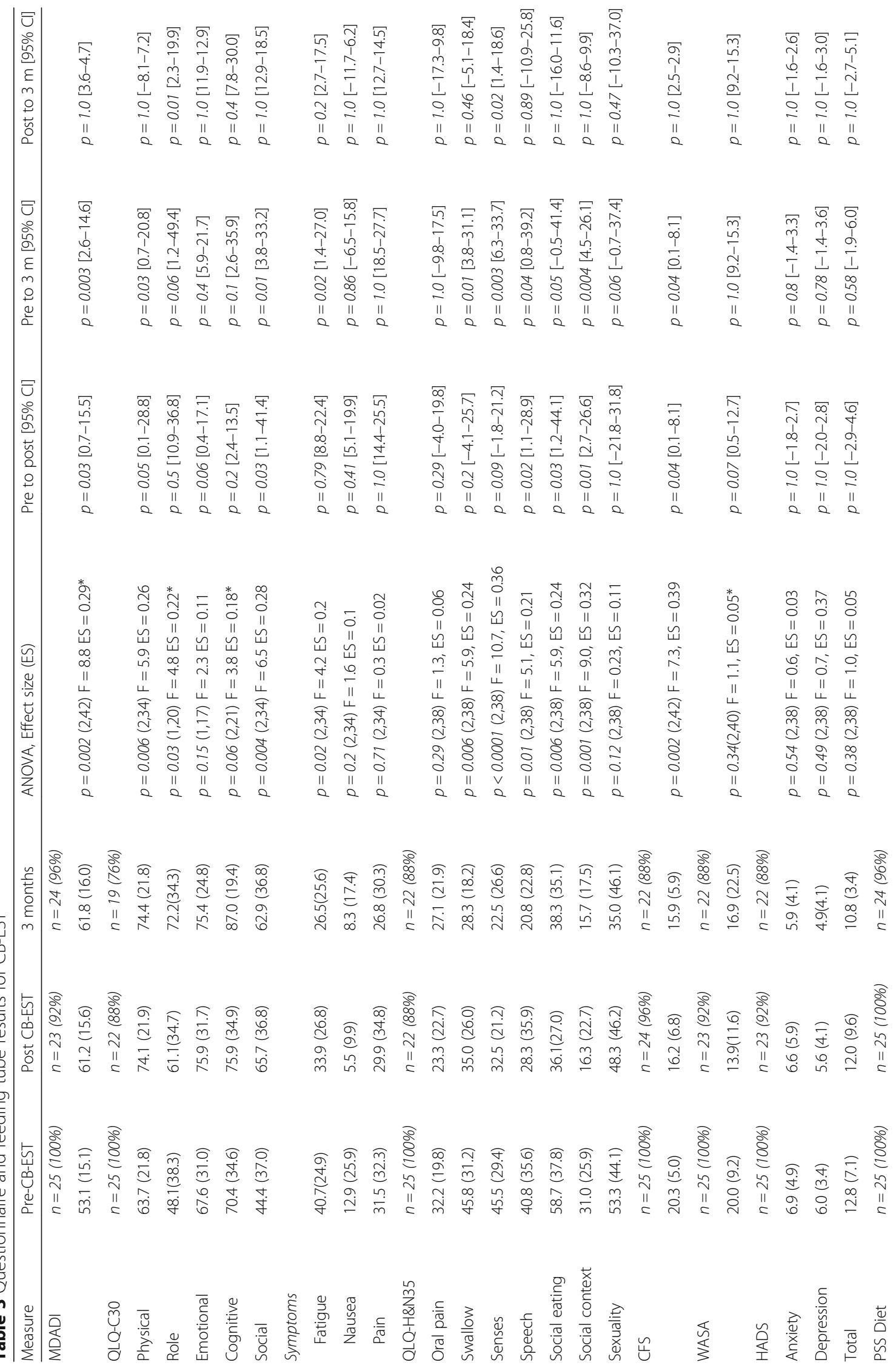




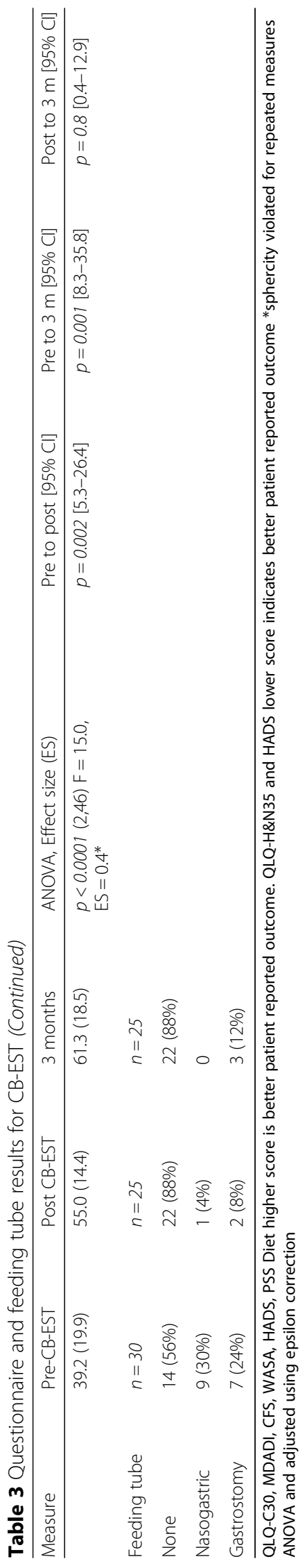


Table 4 Characteristics of interview participants

\begin{tabular}{|c|c|}
\hline \multicolumn{2}{|l|}{ Gender } \\
\hline Male & 9 \\
\hline Female & 3 \\
\hline Age & 61 (range 49-70) \\
\hline \multicolumn{2}{|l|}{ Disease site } \\
\hline Oropharynx & 8 \\
\hline Oral & 1 \\
\hline Nasopharynx & 2 \\
\hline Larynx & 1 \\
\hline \multicolumn{2}{|l|}{ T Stage } \\
\hline 2 & 5 \\
\hline 3 & 5 \\
\hline 4 & 2 \\
\hline \multicolumn{2}{|l|}{ Node stage } \\
\hline 0 & 2 \\
\hline 1 & 3 \\
\hline 2 & 7 \\
\hline \multicolumn{2}{|l|}{ Treatment } \\
\hline Chemoradiotherapy & 9 \\
\hline Surgery and radiotherapy $+/$ - chemotherapy & 3 \\
\hline Time post-treatment (months) & Median 5 range $1-60$ \\
\hline \multicolumn{2}{|l|}{ MDADI pre to post CB-EST } \\
\hline Decreased (4-19 points) & 3 \\
\hline Similar (0-4 points) & 3 \\
\hline Increased (5-36 points) & 6 \\
\hline
\end{tabular}

reluctant, not used to discussing emotional issues, and not the type to require psychological support.

'It will not fit everybody. Some people probably won't want to sit there and say about their life and say how your wife was in tears and say how you were in tears and talk about things like that'(S10 aged 54 years).

However, even those unaccustomed to this approach felt techniques and tasks were tailored to their requirements. In part, due to the ability to tailor the intervention, all participants agreed that there is potential for anyone to benefit from CB-EST. Some said that they didn't know what to expect but either approached sessions with an open mind or simply a 'nothing to lose' attitude. Even those who thought they were less likely to get much out of the sessions reported benefit in interviews, which was not always reflected in their MDADI scores.

\section{Discussion}

CB-EST is a novel treatment and had good rates of patient uptake and retention; formulations and goals were possible for most, candidate measures had good uptake and completeness providing some evidence of effect, and patients reported that they liked the intervention. CBEST was delivered by a CBT trained SLT and was completed within ten sessions. Results need to be interpreted with caution as patients were self-selecting and not consecutively screened and therefore it is likely that more willing patients volunteered for the intervention. Recruitment rates were marginally lower than those reported in other general HNSCC psychosocial interventions [10]. Early indications are that CB-EST is an acceptable intervention for a range of HNSCC patients not confined to treatment type, time posttreatment, or site of disease. The sample was weighted towards patients with advanced staged disease, the majority having combined modality treatment. This was likely due to the predominance of self-reported dysphagia by patients treated with chemoradiotherapy or surgery plus adjuvant radiotherapy [1, 21]. Interview data suggest that people had individual preferences as to how soon CB-EST might be offered following their treatment. Barriers to recruitment were identified. Practicalities of regular out-patient attendance needs to be taken into consideration following intensive HNSCC treatment regimens as well as additional financial costs to the patient [22]. Not all HNSCC patients wish to receive psychological support and may be unsuitable for such an intervention [23]. Drop-out rates due to disease or ill-health are expected but unavoidable in the HNSCC population.

Our previous qualitative work showed that patients view their eating and drinking problems more broadly than just physical impairment [5]. CB-EST was able to respond to individual need, addressing the psychosocial issues of dysphagia by integrating core features of CBT alongside swallowing therapy, with some reporting general improvement in their quality of life. The most common patient goal was removal of feeding tube, which involved a range of physical, cognitive and behavioural techniques. Addressing unhelpful thinking habits was a technique used across all goals. Goals for improving confidence in social eating or adjustment to permanent non-oral feeding did not require impairment based swallowing therapy.

This study employed a selection of candidate measures to assess patient acceptability and sensitivity to change. All achieved a completion rate of $\geq 88 \%$. Outcomes specifically related to eating and drinking (MDADI, EORTC HN35 Swallow and Social Eating and PSS Diet score) were responsive to change, although lack of follow up data from drop outs may positively skew results. Elsewhere, a comparable longitudinal cohort study reported a MDADI mean difference of 4.7 points in the first year post-treatment [24], suggesting some spontaneous adjustment occurs. Under trial conditions for a 
prophylactic swallow exercise intervention, minimal change was seen in EORTC HN35 Swallow and Social Eating scores (mean difference 0 and 5) [25]. The current study found no change on the HADS, despite CBT being an effective intervention for anxiety and depression. This may be due to the sample size, although approximately two thirds of pre-CB-EST patients were either non-cases or had borderline mood issues according to cut-off criteria.

\section{Future work}

This preliminary study directs several areas for further investigation. Future work on refining the selection criteria is required, with early results suggesting that some patients may be more suitable for CB-EST than others. CB-EST was delivered by a single SLT; whether SLTs are willing to be trained, the extent of training and access to regular supervision is unknown. In order to assess effectiveness, CB-EST would need to be protocoled, while allowing for an individually tailored approach, with fidelity checks. A proportion of patients declined participation, citing difficulties with regular attendance. It is uncertain as to whether CB-EST requires face to face intervention or if components could be administered via other mediums such as telemedicine, self-help booklets or web-based programmes. However, patients often express a preference for individual, face to face help, preferably at home [23]. It is unknown as to whether patients would be willing to be randomised in the context of a trial. Using MDADI scores, a sample size for a future trial would require 84 patients, providing $80 \%$ probability of detecting a difference at two sided significance level of 0.05. Accounting for an intervention completion rate of $58 \%$ from the available sample, 145 patients would be required to conduct such a study.

\section{Conclusion}

The addition of cognitive behavioural techniques to swallowing therapy delivered by a trained SLT, is a feasible and acceptable treatment, addressing the physical and psychosocial components of HNSCC dysphagia. Further work is needed to establish efficacy, effectiveness and cost-effectiveness of the intervention, in the context of a randomised controlled trial.

\footnotetext{
Abbreviations

CB-EST: Cognitive Behavioural-Enhanced Swallow Therapy; CBT: Cognitive Behaviour Therapy; CFQ: Chalder Fatigue Questionnaire; EORTC-

HN: European Organization for Research and Treatment of Cancer - Head and Neck module; EORTC-QLQ: European Organization for Research and Treatment of Cancer- Quality of Life Questionnaire; HADS: Hospital Anxiety and Depression Scale; HNSCC: Head and neck squamous cell carcinoma; MDADI: MD Anderson Dysphagia Inventory; NGT: Nasogastric tube; PSS: Performance Status Scale; SLT: Speech and Language therapist; WASA: Work and Social Adjustment Scale
}

\section{Acknowledgments}

There are no other acknowledgments.

\section{Funding}

Dr. J Patterson is funded by a National Institute for Health Research award (Clinical Lecturer). This paper presents independent research funded by the National Institute for Health Research. The views expressed are those of the authors and not necessarily those of the NHS, the NIHR or the Department of Health. The funding body peer reviewed the study, but had no role in the design, data collection and analysis, interpretation of data or writing of the manuscript. The authors declare no conflicts of interest.

\section{Availability of data and materials}

Datasets analysed during the current study are available from the corresponding author on reasonable request. All data analysed during this study are included in this published article.

\section{Authors' contributions}

All authors made substantial contributions to the conception and design of this study. JP, EMC, CE and VD conceived the research design and developed the study protocol. JP delivered the intervention and VD provided supervision. JP acquired and analysed the quantitative data. MF and MB acquired and analysed the qualitative data. All authors were involved in the interpretation of data and preparation of the manuscript. They have revised the content, are accountable and have given approval for the current submission to be considered for publication.

\section{Ethics approval and consent to participate}

Ethical approval was granted by the UK North East Research Ethics Committee reference 14/NE/1045. All patients gave written informed consent prior to participation.

\section{Consent for publication}

Not applicable.

\section{Competing interests}

The authors declare that they have no competing interests.

\section{Publisher's Note}

Springer Nature remains neutral with regard to jurisdictional claims in published maps and institutional affiliations.

\section{Author details}

${ }^{1}$ Institute of Health and Society, Newcastle University, The Baddiley-Clark Building, Richardson Road, Newcastle upon Tyne NE2 4AX, UK. ${ }^{2}$ Speech \& Language Therapy Department, Sunderland Royal Hospital, Sunderland, UK. ${ }^{3}$ Faculty of Health and Life Sciences, Northumbria University, Newcastle upon Tyne, UK. ${ }^{4}$ Psychology Department, Northumbria University, Newcastle upon Tyne, UK.

Received: 1 March 2017 Accepted: 8 December 2017

Published online: 02 January 2018

\section{References}

1. Wilson JA, Carding PN, Patterson JM. Dysphagia after nonsurgical head and neck cancer treatment: patients' perspectives. Otolaryngology - Head \& Neck Surgery. 2011;145(5):767-71.

2. Semenov YR, Starmer HM, Gourin CG. The effect of pneumonia on shortterm outcomes and cost of care after head and neck cancer surgery. Laryngoscope. 2012;122(9):1994-2004.

3. Ichikura K, Yamashita A, Sugimoto T, Kishimoto S, Matsushima E. Persistence of psychological distress and correlated factors among patients with head and neck cancer. Palliative \& Supportive Care. 2016;14(1):42-51.

4. Lin BM, Starmer HM, Gourin CG. The relationship between depressive symptoms, quality of life, and swallowing function in head and neck cancer patients 1 year after definitive therapy. Laryngoscope. 2012;122(7):1518-25.

5. Patterson JM, McColl E, Wilson J, Carding P, Rapley T. Head and neck cancer patients' perceptions of swallowing following chemoradiotherapy. Support Care Cancer. 2015;

6. Cousins N, MacAulay F, Lang H, MacGillivray S, Wells M. A systematic review of interventions for eating and drinking problems following treatment for 
head and neck cancer suggests a need to look beyond swallowing and trismus. Oral Oncol. 2013;49(5):387-400.

7. Perry A, Lee SH, Cotton S, Kennedy C. Therapeutic exercises for affecting post-treatment swallowing in people treated for advanced-stage head and neck cancers. Cochrane Database Syst Rev. 2016;16(8):CD011112.

8. Reich M, Leemans CR, Vermorken JB, Bernier J, Licitra L, Parmar S, Golusinski W, Lefebvre JL. Best practices in the management of the psycho-oncologic aspects of head and neck cancer patients: recommendations from the European head and neck cancer society make sense campaign. Ann Oncol. 2014;25(11):2115-24.

9. Semple C, Parahoo K, Norman A, McCaughan E, Humphris G, Mills M. Psychosocial interventions for patients with head and neck cancer. Cochrane Database Syst Rev. 2013;

10. Semple CJ, Dunwoody L, Kernohan WG, McCaughan E. Development and evaluation of a problem-focused psychosocial intervention for patients with head and neck cancer. Support Care Cancer. 2009;17(4):379-88.

11. Miller T, Deary V, Patterson J. Improving access to psychological therapies in voice disorders: a cognitive behavioural therapy model. Current Opinion in Otolaryngology and Head and Neck Surgery. 2014;22(3):201-5.

12. Eldridge SM, Chan CL, Campbell MJ, Bond CM, Hopewell S, Thabane L, Lancaster GA. CONSORT 2010 statement: extension to randomised pilot and feasibility trials. BMJ. 2016;355

13. Chen AY, Frankowshi R, Bishop-Leone J, Hebert T, Leyk S, Lewin J, Goepfert $H$. The development and validation of a dysphagia-specific quality-of-life questionnaire for patients with head and neck cancer: the M. D. Anderson dysphagia inventory. Archives of Otolaryngol Head Neck Surg. 2001;127(7):870-6

14. Lancaster GA, Dodd S, Williamson PR. Design and analysis of pilot studies: recommendations for good practice. J Eval Clin Pract. 2004;10(2):307-12.

15. Hutcheson KA, Barrow MP, Lisec A, Barringer DA, Gries K, Lewin JS. What is a clinically relevant difference in MDADI scores between groups of head and neck cancer patients? Laryngoscope. 2015;

16. Bjordal K, de Graeff A, Fayers PM, Hammerlid E, van Pottelsberghe C, Curran D, Ahlner-Elmqvist M, Maher EJ, Meyza JW, Bredart A, et al. A 12 country field study of the EORTC QLQ-C30 (version 3.0) and the head and neck cancer specific module (EORTC QLQ-H\&N35) in head and neck patients. Eur J Cancer. 2000;36(14):1796-807.

17. Chalder T, Berelowitz G, Pawlikowska T, Watts L, Wessely S, Wright D, Wallace EP. Development of a fatigue scale. J Psychosom Res. 1993;37(2):147-53.

18. Mundt JC, Marks IM, Shear MK, Greist JM. The work and social adjustment scale: a simple measure of impairment in functioning. Br I Psychiatry. 2002;180(5):461-4.

19. Zigmond AS, Snaith RP. The hospital anxiety and depression scale. Acta Psychiatr Scand. 1983;67(6):361-70.

20. List MA, Mumby P, Haraf D, Siston A, Mick R, MacCracken E, Vokes E. Performance and quality of life outcome in patients completing concomitant chemoradiotherapy protocols for head and neck cancer. Qual Life Res. 1997;6(3):274-84.

21. Mittal BB, Pauloski BR, Haraf DJ, Pelzer HJ, Argiris A, Vokes EE, Rademaker A, Logemann JA. Swallowing dysfunction - preventative and rehabilitation strategies in patients with head-and-neck cancers treated with surgery, radiotherapy, and chemotherapy: a critical review. International Journal of Radiation Oncology Biology Physics. 2003;57(5):1219-30.

22. Villa C, Meregaglia M, Rognogni C, Pistillo P, Orlandi E, lacovelli A, Granata R, Alfieri S, Bergamini C, Resteghini $C$, et al. Health and economic outcomes of two different follow up strategies in effectively cured advanced head and neck cancer patients. Annals of Oncology. 2016;27(suppl_4):iv87.

23. Richardson AE, Morton R, Broadbent E. Psychological support needs of patients with head and neck cancer and their caregivers: a qualitative study. Psychol Health. 2015;30(11):1288-305.

24. Roe JWG, Drinnan MJ, Carding PN, Harrington KJ, Nutting CM. Patientreported outcomes following parotid-sparing intensity-modulated radiotherapy for head and neck cancer. How important is dysphagia? Oral Oncol. 2014;50(12):1182-7.

25. Mortensen HR, Jensen K, Aksglæde K, Lambertsen K, Eriksen E, Grau C. Prophylactic swallowing exercises in head and neck cancer radiotherapy. Dysphagia. 2015;

\section{Submit your next manuscript to BioMed Central and we will help you at every step:}

- We accept pre-submission inquiries

- Our selector tool helps you to find the most relevant journal

- We provide round the clock customer support

- Convenient online submission

- Thorough peer review

- Inclusion in PubMed and all major indexing services

- Maximum visibility for your research

Submit your manuscript at www.biomedcentral.com/submit
Biomed Central 